\title{
Lidil
}

Revue de linguistique et de didactique des langues

\section{Rapport de stage et Mémoire professionnel entre normes et représentations}

Anne-Catherine Oudart et Marie-Renée Verspieren

\section{(2) OpenEdition}

1 Journals

Édition électronique

URL : http://journals.openedition.org/lidil/16

DOI : $10.4000 /$ lidil. 16

ISSN : 1960-6052

Éditeur

UGA Éditions/Université Grenoble Alpes

Édition imprimée

Date de publication : 1 décembre 2006

ISBN : 2-914176-15-5

ISSN : $1146-6480$

\section{Référence électronique}

Anne-Catherine Oudart et Marie-Renée Verspieren, « Rapport de stage et Mémoire professionnel entre normes et représentations », Lidil [En ligne], 34 | 2006, mis en ligne le 07 avril 2008, consulté le 10 décembre 2020. URL : http://journals.openedition.org/lidil/16 ; DOI : https://doi.org/10.4000/lidil.16

Ce document a été généré automatiquement le 10 décembre 2020.

(c) Lidil 


\title{
Rapport de stage et Mémoire professionnel entre normes et représentations
}

\author{
Anne-Catherine Oudart et Marie-Renée Verspieren
}

1 La question de l'accompagnement des étudiants dans l'écriture de rapports de stage et/ou de mémoires professionnels est un sujet qui entraine très souvent des débats animés et contrastés chez les enseignants : comme si le simple fait d'évoquer ces activités d'écriture réveillait des conceptions individuelles bien ancrées et non révisables. Chacun s'évertuant à convaincre autrui que le "produit fini ", auquel il se réfère, correspond à l'image de l'objet attendu. Il y aurait ainsi des représentations qui conduisent nos stratégies d'accompagnement et qui nous permettent d'apposer, lors des évaluations finales de soutenance, des annotations appréciatives, précises. Force est de constater que, lors des délibérations entre les membres des jurys, des critères d'évaluation prédominants se révèlent.

2 D'un autre côté, l'inquiétude perceptible et le désarroi récurrent des étudiants face à ces écrits longs nous révèlent toutes les ambigüités et les messages paradoxaux que nous véhiculons en cherchant à articuler à la fois des préconisations " aidantes ", en lien avec des ouvrages didactiques et des conceptions « ouvertes» teintées de nos représentations. C'est dans ce dédale de consignes et de conseils que les étudiants se débattent, cherchant tantôt à se rassurer à travers des normes explicites, ou cherchant à élaborer un « produit idéal » conforme à l'image qu'ils se sont construite des genres « rapport de stage » et «mémoire professionnel », au plus près des exigences évaluatives de l'activité d'écriture.

Ces remarques préliminaires nous ont conduites, dans le cadre de cet article, à nous poser la question transversale suivante : que savons-nous des normes et des représentations qui conduisent les pratiques scripturales des étudiants? De cette interrogation centrale, il nous a semblé que d'autres questions pouvaient découler: ces normes sont-elles réinvesties par les étudiants? Comment sont-elles intégrées dans nos pratiques évaluatives? Y a-t-il un socle commun sur lequel nous nous entendons? En quoi l'activité 
d'écriture « rapport de stage et mémoire professionnel » est-elle porteuse de messages paradoxaux?

4 Pour apporter un éclairage à ce questionnement, nous avons choisi de croiser trois corpus distincts, en trois temps; d'abord, nous recueillerons les préconisations d'ouvrages évoquant les définitions des genres « rapports de stage » et "mémoires professionnels » (une dizaine d'ouvrages et/ou de sites Internet), notre objectif étant ici de chercher ces normes explicites qui ont pu construire les représentations des uns et des autres. Ensuite, nous examinerons leurs mises en œuvre dans l'écriture, et leurs places dans l'évaluation. Pour cela, nous avons choisi, dans un premier temps, de nous appuyer sur une pratique d'écriture, celle du rapport de «stage ouvrier » effectué en fin de première année dans une école d'ingénieur en agro-alimentaire (40 rapports). Il s'agit d'un écrit qui affiche clairement des intentions expérientielles et questionne de ce fait le genre "rapport de stage »: s'agit-il d'un témoignage, d'un récit vécu, d'une observation simple, d'une situation de travail ? Dans un deuxième temps, nous avons choisi de nous pencher sur une autre pratique d'écriture, celle du mémoire professionnel d'une licence IUP des « Métiers de la formation » et, plus précisément, sur les commentaires appréciatifs justifiant la note accordée à cet écrit (200 commentaires).

5 En montrant que les normes et les représentations de ces deux genres conduisent les activités d'écriture des apprenants et qu'elles contribuent à orienter les pratiques évaluatives, nous souhaitons faire émerger les zones de tension probables, voire les paradoxes auxquels sont confrontés les étudiants dans l'élaboration de leurs productions écrites et les enseignants dans l'accompagnement de ces productions. Des pistes didactiques, inhérentes à ces constats, pourront se dégager.

Les normes explicites : les préconisations des ouvrages de référence

6 Notre corpus porte sur des ouvrages mis à la disposition des étudiants en bibliothèque universitaire et sur des sites Internet, consacrés aux mémoires professionnels et/ou mémoires et rapports de stage. Nous ferons état ici de quelques-unes de nos observations. Tout d'abord, le mémoire professionnel est souvent défini en opposition au rapport de stage. "Le mémoire professionnel, même s'il s'appuie sur une activité professionnelle, n'est ni un rapport de stage ou d'activité, ni un dossier d'enquête et d'observation " (Maffre, 1998 : 23) ; « un mémoire n'est pas un rapport de stage » (Guigue, 1995 : 34). On trouve une catégorisation de type "genre second » et « genre premier ». Les connecteurs tandis que, alors que, à la différence de, etc. donnent d'ailleurs très clairement des indicateurs appréciatifs. Si le rapport de stage se distingue du compte rendu en ce « qu'il fait plus que rassembler des informations sur un lieu ou une situation " (Laborde-Milaa, 1999 : 4), il reste néanmoins un "genre second " par rapport au mémoire professionnel, pour la plupart des auteurs: "À la différence du rapport de stage, le mémoire professionnel n'est pas constitué essentiellement par l'analyse d'une expérience pratique, il doit être basé sur une réflexion théorique...' ". Non cantonné à des frontières strictes, le mémoire professionnel est perçu comme un "genre premier » : "À la différence d'un rapport de stage, le mémoire professionnel déborde la situation pédagogique parce qu'il ne se limite pas à assurer ou prouver un ensemble d'apprentissage » (Guigue, 1995 : 34). Certes, l'alliance "réflexion et action » est évoquée pour les deux écrits, «le rapport de stage restitue une expérience en milieu professionnel, tout en manifestant une réflexion distanciée »(Laborde-Milaa, 1999: 4), mais le mémoire professionnel est cependant présenté comme un écrit devant davantage questionner la réalité, l'analyser et la transformer : «Le mémoire professionnel témoigne d'une capacité à agir, à réfléchir et à 
analyser son action en vue de l'améliorer » (Maffre, 1998: 13). C'est en ce sens que la circulaire ministérielle (circulaire 91-2002 du 2 juillet 1991) définit le mémoire professionnel d'IUFM de la façon suivante : «Il ne doit ni constituer une simple narration d'un travail personnel sans analyse critique, ni être une réflexion théorique ou historique extérieure à l'expérience du professeur stagiaire ». Les verbes employés par les ouvrages pour évoquer le rôle du mémoire professionnel se situent plutôt dans un registre " réflexif » : étudier/analyser/comprendre. Alors que ceux du rapport de stage sont dans un registre "narratif » : exposer/ expliquer/ rendre compte/ décrire/raconter/restituer.

7 La distinction porte donc principalement sur la notion de prise de distance ou de prise de hauteur: «Le mémoire professionnel montre que vous êtes capable de prendre de la distance critique et de la hauteur de vue par rapport à une pratique quotidienne, que vous savez en rendre compte par écrit et que tout cela vous donne une plus grande maitrise de votre métier» (Maffre, 1998: 33). Parce qu'il est souvent associé à des missions ou actions qui nécessitent des phases de conception, de réalisation et d'analyse, «il exige une part de théorisation» (Laborde-Milaa, 1999: 4). Véritable "outil de professionnalisation» (L'Heudé, 2005: 298), le mémoire professionnel est aussi «un moyen de se faire reconnaitre par ses pairs " (Maffre, 1998: 33). Le rapport de stage est, lui, présenté davantage comme un écrit qui rend compte d'une réalité observable : «il s'agit de la description de toutes les tâches effectuées ».

8 Ces approches veulent figer des modèles. Néanmoins, ceux-ci varient parfois selon les classifications opérées par les ouvrages. En effet, pour certains, il existe différents types de mémoire et différents types de rapport de stage. Par exemple, Myriam Greuter (2001: 11) classe les rapports de stage en trois catégories : rapport de stage simple/rapport de recherche/rapport de synthèse ; et Bruno Camus (1998:7) parle de rapport d'expérience/ rapport de recherche/rapport de compilation. Certains auteurs évoquent même le terme "mémoire de terrain ». On voit nettement que les appellations sont liées au rôle et à la place que l'on accorde au stage, objet de la mission ou à la commande. Isabelle LabordeMilaa (1999: 4) évoque d'ailleurs très clairement l'incidence du type de stage sur le type d'écrit et catégorise les stages en trois grandes familles: "stage de découverte, stage d'application, stage de mission ou d'action ». Ces catégorisations montrent, comme le suggère Rozenn Guibert (2003 : 93), que « la notion de genre est à relier étroitement aux situations de communication, aux enjeux et aux stratégies des acteurs ».

D'un côté, les auteurs cherchent à figer un modèle pour en faire un objet d'enseignement. Ils semblent s'accorder plutôt sur une modélisation de type : le compte-rendu (j'ai vu) ; le rapport de stage (j'ai vu, j'ai compris) et le mémoire professionnel (j'ai vu, j'ai lu, j'ai analysé, j'ai compris, je propose et, éventuellement, j'évalue ma/mes propositisons). Ils affirment l'existence de normes d'écriture formelle: normes de présentation, prise en compte du destinataire, critères de lisibilité, utilisation de plans stratégiques (plus particulièrement pour les rapports de stage). D'un autre côté, ils cherchent à ne pas enfermer ces genres dans une configuration réductrice et laissent parfois un flou quant à la finalisation du produit écrit.

10 Que ce soit le rapport de stage ou le mémoire professionnel, ces écrits prennent appui sur une activité qui a été effectuée et qui a valeur d'expérience professionnelle: ils permettent tous les deux de «faire le point sur un métier » (Laborde Milaa, 1999: 23), c'est d'ailleurs ce qui justifie le fait de les réunir pour notre étude. Alors que le stage parait déterminant sur le "genre » à produire, son lien avec la restitution dans l'écrit reste néanmoins peu abordé par les auteurs (Laborde Milaa, 1999: 34). En d'autres 
termes, comment rendre compte d'un stage inintéressant? Faut-il «parler des journées creuses?» (Laborde-Milaa, 1999 : 34). Comment évoquer un stage peu positif? À ce sujet, les auteurs restent souvent silencieux : «Si le stage se passe mal, vous devrez affronter la situation seul [...] on ne peut malheureusement plus faire marche arrière. Il faut faire avec.» (Greuter, 2001: 68), ou pour le moins ambigus : «l'étudiant sera évalué sur le choix de son stage, sur ses conditions d'obtention, sur les activités qu'il a menées, sur son enrichissement personnel et éventuellement sur sa contribution à l'entreprise, l'organisme ou l'institution qui l'a accueilli. » (Camus, 1998: 16). De ce fait, comment distinguer l'évaluation du stage et l'évaluation de la production écrite restituant l'expérience.

11 Nous verrons notamment, dans la troisième partie, que les pratiques évaluatives pointent cette ambigüité, et que les commentaires associés aux évaluations amalgament souvent ce que Sylvie l'Heudé (2005: 301) appelle les évaluations d'ordre de l'hétéroréférentiel (évaluation dans un système normé) et de l'ordre de l'autoréférentiel (évaluation d'un processus de professionnalisation).

Les représentations du rapport de stage dans les pratiques rédactionnelles : le cas du rapport de stage ouvrier en école d'ingénieur

L'analyse de plusieurs rapports de stage rédigés par des ingénieurs en agro-alimentaire en $1^{\text {re }}$ année va nous permettre de comprendre comment ces préconisations sont réinvesties dans les pratiques rédactionnelles. À la suite d'un stage d'un mois en tant qu'ouvrier, les étudiants doivent rédiger un rapport de stage qui donnera lieu à une soutenance. Ce stage a pour objectif de donner l'occasion aux étudiants d'avoir un contact avec les gestes du métier d'ouvrier: activités répétitives, travail à la chaine, travail posté, etc. L'analyse des productions écrites par les étudiants montre très nettement une restitution de l'expérience à travers l'utilisation de normes figées, dans une construction formelle en trois parties : on pose le cadre (lieu, entreprise), on décrit l'activité effectuée, on observe les relations humaines et le management de proximité.

Cette démarche met en évidence deux façons d'aborder le rapport de stage :

- par le schéma général de l'action à travers une logique fonctionnelle. Par exemple, Isabelle décrit son activité de manutentionnaire : « remplir les chariots avec les baguettes, suivre la mise sous film, les porter sur des palettes, vérifier l'emballage, les déposer sur un plateau, etc. »;

- par l'exposition d'une activité à travers ses affects, ses valeurs : «J'ai découvert que l'esprit d'équipe et la communication sont des outils essentiels pour une efficacité maximale »; « une chose est claire, il faut aller vite !». Les étudiants font émerger les aspects émotionnels dans l'action sans aller au-delà, se contentant d'exposer le plus souvent $\mathrm{du}$ ressenti, du vécu.

14 Après le corps du mémoire, la conclusion amorce des aspects analytiques qui permettent à l'étudiant de faire ressortir deux points forts. Le premier est de révéler l'indicible de ce qui finalement a forgé les premières représentations fortes du champ professionnel. Élodie termine ainsi son rapport : «Ce stage m'a fait prendre conscience de la pression de résultat qui pèse en permanence sur les ingénieurs ». Enfin, Hervé se lance en toute fin de rapport dans une critique des modes de management: «J'ai constaté que le personnel ouvrier recevait de très nombreuses consignes, mais elles sont noyées dans un ensemble d'informations pas toujours adaptées à la totalité du personnel et à leur objectif de travail ». 

là que s'arrête la mission du rapport de stage : «évoquer » n'est pas "prouver ». Le recueil de données servant à l'analyse reste finalement succinct, issu d'impressions subjectives, glanées parfois aux détours de conversations conviviales, informelles. C'est d'ailleurs ce que révèle Virginie lorsqu'elle écrit : «C'est fou ce que l'on apprend comme choses pendant les pauses! ».

Mais s'autoriser à penser, c'est s'autoriser à prendre du recul sur le métier, c'est aussi prendre le risque de se retrouver en dissonance cognitive ou de dépasser sa posture de stagiaire. Ainsi, Pierre écrit dans sa conclusion : «Quel peut être l'avenir d'une entreprise où les contrats de travail se terminent et ne sont plus renouvelés et où la fonction d'atelier relais prime sur la fabrication et donc sur la vente des produits de la ferme, premier revenu de l'entreprise?». La question posée ici devient une précaution rhétorique qui permet de dire sans s'engager. C'est bien la question de la posture du stagiaire, à la vision partielle, subjective et fugitive, qui est en jeu. La mission de l'étudiant ici n'est pas de chercher à tout comprendre mais bien d'appréhender un métier dans sa dimension gestuelle, dans une perspective d'insertion professionnelle.

Le deuxième objectif de la partie conclusive est de révéler à l'évaluateur la façon dont l'étudiant s'est immergé dans le milieu professionnel, et comment il s'y est intégré : «L'intégration à l'équipe a été très facile »; « très vite, j'ai fait partie de l'équipe »; « mon intégration s'est faite sans difficulté en quelques jours. On m'a tout de suite expliqué le fonctionnement général. Tous ont été disponibles pour répondre à mes questions ». Les termes employés dans ces conclusions montrent que les étudiants souhaitent :

- laisser une trace positive de leur immersion professionnelle ;

- fournir aux enseignants des indications leur permettant d'accorder une valeur au stage et à leur future fonction managériale.

En effet, l'activité d'ouvrier est observée du point de vue de la posture d'ingénieur : « Il m'a donc paru essentiel, en tant que futur ingénieur, de réussir à communiquer avec l'ensemble des salariés ». D'une certaine façon, le «stage ouvrier » n'est pas vécu comme expérience pour devenir ouvrier, mais bien comme expérience pour devenir cadre : « Ce stage me conforte dans l'idée que le métier d'ingénieur est à la fois basé sur une formation scientifique mais aussi sur une capacité à être sociable et attentif à l'humain ». Il est alors révélateur des qualités individuelles nécessaires pour exercer non pas l'activité faite, mais bien celle que potentiellement il sera amené à faire: «j'ai pris conscience que le respect est une des clés pour la réussite d'un travail en équipe »; «l'humilité et l'écoute seront, à mon sens, les qualités demandées à un jeune ingénieur »; «j'ai retenu qu'en tant qu'ingénieur il faut sans cesse apprendre de nouvelles techniques, se remettre en question et être à l'écoute de ses collègues ». Regarder l'activité effectuée par le prisme de son futur métier de cadre introduit des changements de posture, autorisant des réflexions dans une mise en perspective professionnelle différente. «J'ai appris que je préfère avoir des responsabilités et entreprendre des projets plutôt que de rester toute la journée devant une machine ». Venus en tant que stagiaires ouvriers, c'est en tant que futurs cadres qu'ils regardent l'entreprise. Les propos tenus en témoignent: «J'ai appris que la communication intra personnelle est fondamentale pour le métier d'ingénieur ». Ils en tirent même des enseignements pour leur avenir professionnel, comme pour leur activité managériale : «J'ai également pu comprendre l'importance de prouver à chaque employé que son travail est essentiel pour le bon fonctionnement de l'entreprise ». 

note des mémoires professionnels. Ces annotations, rédigées sur un support prévu à cet effet, sont restituées oralement à l'étudiant le jour de la soutenance. Nous avons examiné attentivement plus de deux cents annotations provenant de dix enseignants chargés d'évaluer les mémoires professionnels de licencé2. Par cette investigation, nous voulions savoir comment les normes attendues étaient, ou éventuellement n'étaient pas, prises en compte dans les annotations et ce que cela pouvait révéler des représentations des évaluateurs. enseignants, nous avons cherché les indicateurs appréciatifs qui fondent la note. Dix items sont alors apparus: la prise de distance, la conceptualisation, la méthodologie, l'implication, la conviction, la qualité et l'intérêt du stage, le formalisme de l'écrit, les références aux exigences de l'écrit universitaire, l'inscription des propos dans une trajectoire. Nous avons ensuite cherché à savoir si les évaluateurs accordaient la même valeur à ces items. À cet effet, nous avons choisi de les hiérarchiser en tenant compte de leur fréquence d'apparition en lien avec la note attribuée. Les résultats ont très clairement fait apparaitre l'existence de trois niveaux de critères: les critères « incontournables », autrement dit déterminants pour la qualité supposée du mémoire ; les critères « recherchés », c'est-à-dire largement plébiscités par les évaluateurs ; et enfin les critères «souhaités ", qui mettent juste une plus value à la note. Voici le classement obtenu : trajectoire professionnelle.

Les critères incontournables 
bonne distance par rapport à l'action", ainsi que "la capacité à se remettre en question ». On retrouve la définition de Françoise Cros (1994: 107-108) lorsqu'elle parle, à propos du mémoire professionnel, d'un travail de questionnement et de mise à distance permettant d'analyser des réalités de terrain. Il s'agit d'aller au-delà de la première impression, et de produire des connaissances qui ne seraient pas accessibles sans cette attitude réflexive. De ce fait, on comprend pourquoi le manque de prise de recul donne lieu à des commentaires dépréciatifs: "faiblesse de la réflexion"; "synthèse qui pourrait décoller du terrain et élargir la réflexion »; « nécessité de plus d'ouverture et de recul ».

Dans cette prise de recul, la capacité à relier action-réflexion, théorie et pratique est fondamentale. Elle est associée aux lectures théoriques: «dommage que les propos ne soient pas davantage relayés par des lectures théoriques qui auraient donné ainsi un peu de distance au vécu ressenti ». Les apports théoriques et conceptuels sont le ciment de la réflexion, mais ne doivent être «ni trop présents ni trop plaqués». Cette «bonne mobilisation des ressources " permet la prise de recul dans l'action: «il faut lire pour prendre du recul et éclairer l'action ». C'est dans un "rapport très instrumental aux théories que se construisent les mémoires professionnels » (Chaix et Lemey, 1994: 15); il s'agit, pour les étudiants, de tirer des lectures une idée, un concept qui leur parlent, et qu'ils s'approprient à leur manière.

La nécessité d'éclairer l'action est récurrente dans les propos des membres du jury qui, d'ailleurs, mentionnent le recueil de données comme « outil permettant d'interroger la pratique». Le recours à une méthodologie («bon travail d'observation; bonne documentation ») est largement encouragé, car elle permet d'éviter les «jugements non étayés ». Lectures et recueils de données nourrissent la réflexion et sont ensuite «à réexploiter dans une pensée structurée ».

Les critères recherchés

Dans cette catégorie, nous citerons les critères qui donnent des indications de professionnalisation comme l'implication dans l'activité, la conviction de l'écrivant et l'intérêt de l'activité.

31 D'une certaine façon, c'est la qualité de la posture, à la fois dans l'écrit et dans l'action, qui est recherchée: «forte implication dans le travail»; «réflexion d'un acteur engagé »; " est passée d'une posture d'enseignante à celle d'une formatrice d'adulte »; " a dépassé ses a priori de départ ». On remarque la présence de commentaires incitant à la fois à avoir des convictions personnelles et à savoir les pondérer, encourageant à s'impliquer tout en restant distancié : « avis nuancé »; " travail qui pondère avec succès ses convictions personnelles »; « attention aux jugements catégoriques ».

L'implication dans l'action professionnelle facilite une écriture engagée, laquelle a force de conviction : « essaie de nous faire partager ses envies de changement » ; "écrit engagé, convaincant ». Il peut en découler un écrit « enthousiaste »; « positif »; « personnalisé »; " honnête »; « authentique »; « créatif »; « original ». Autant de qualificatifs utilisés par les correcteurs pour apprécier le mémoire.

Enfin, c'est autour de l'activité dans le stage que porte une grande majorité de commentaires. C'est la fonction de production de savoirs professionnels qui est alors recherchée. Écrire, dans le cadre du mémoire professionnel, sert principalement à produire de la connaissance sur l'activité. C'est pourquoi l'utilité du travail effectué et les compétences mobilisées dans l'action sont des indicateurs pris en compte par les jurys: 
« action qui témoigne d'une réelle compétence de formatrice de terrain »; « mémoire qui montre des compétences professionnelles indéniables dans le domaine de l'enseignement des langues ». La qualité de l'activité effectuée, son côté « innovant ", "opérationnel », «pragmatique» met l'expérience professionnelle au cœur de l'évaluation. Tout cela s'inscrit dans une trajectoire professionnelle, laquelle peut d'ailleurs être encouragée : " année charnière positive "; " en tirer des enseignements dans une trajectoire de formation » : « seuil de professionnalisation atteint ». C'est ce que Sylvie L'Heudé (2005: 301) appelle l'évaluation de l'ordre de l'autoréférentiel. C'est-à-dire qu'ici est prise en compte la personne dans son cheminement, son engagement professionnel et son évolution vers sa professionnalisation. Le jury oscille alors entre mettre en avant la valeur qualitative de l'étudiant sur le terrain et la valeur scripturale du mémoire proprement dit.

Les critères souhaités

Ce sont les critères formels qui ont trait d'abord à l'écriture proprement dite: "écrit construit et écriture fluide »; "clarté des propos et rigueur du plan»; "écrit soigné, clair, bien rédigé ", mais également aux exigences liées aux écrits universitaires : « travail qui va au-delà du niveau de la licence "; "écrit ne correspondant pas au cahier des charges universitaires ». Les remarques relatives «à la structuration»; «à l'articulation "; "à la construction »; "à la cohérence »; « au plaisir de lire » et " au souci du lecteur » montrent l'intérêt accordé aux critères de lisibilité. A contrario, la forme peut déplaire : « la qualité très moyenne de l'écrit ne fait pas apparaitre la richesse de la production»; «manque de soin»; «mémoire décousu»; «orthographe!»; «structuration et expression formelle à parfaire ». Il s'agit ici de rappeler à l'étudiant les normes d'écriture, afin qu'il puisse se corriger dans une perspective professionnelle et universitaire.

Ces critères, clairement exprimés par les évaluateurs, confirment bien l'existence de normes et de représentations sous-jacentes qui conduisent l'activité évaluative du genre «mémoire professionnel ». Même si ces trois critères sont reconnus comme importants pour la qualité du mémoire, ils n'ont pas la même portée, selon les évaluateurs. De ce fait, on retrouve des annotations qui révèlent des divergences dans les pratiques évaluatives.

On peut donc penser que l'apprenant a été confronté lors de l'accompagnement de son mémoire à des niveaux d'exigence divergents, lesquels ont pu générer des tensions dans le processus d'écriture. C'est à cet aspect que nous consacrerons notre dernière partie.

Les zones de tension

37 Rapports de stage et mémoires professionnels sont, comme nous venons de le voir, des productions écrites proposées pour témoigner d'une expérience professionnelle. Ils se situent à l'interface d'un monde de normes explicites transmises encore et encore par de multiples ouvrages de référence et d'un monde implicite, celui des représentations des auteurs et des lecteurs. Parfois enseignés dans certains dispositifs, ces écrits sont, en tous les cas, toujours évalués. Leur fonction dans l'évaluation de la professionnalisation d'étudiants-stagiaires leur confère un rôle déterminant, mais, en même temps, ces écrits sont au cœur de tensions à la fois pour l'évaluateur et pour l'écrivant. Pourquoi parler de tension en évoquant la mise en œuvre de ces deux genres? Qu'est-ce qui, dans le processus d'écriture, provoque ces mises en tension?

Pour l'évaluateur, la tension provient de cette nécessité d'évaluer à la fois un produit fini, preuve d'une capacité à construire une pensée, à formaliser un écrit mais aussi d'évaluer un processus d'apprentissage, preuve d'une démarche de construction et d'immersion 
professionnelle. Certains évoqueraient à ce sujet la dichotomie "fond et forme ». Nous préférons, quant à nous, parler d'approches de formalisation d'un écrit professionnel ou de démarches d'appropriation expérientielle relatées dans un écrit professionnalisant.

Les étudiants, d'ailleurs, perçoivent bien que, selon l'approche privilégiée par l'accompagnateur, leurs stratégies discursives ont intérêt à différer : mettre l'accent sur «le dire » et donc la formalisation, ou se concentrer sur « le faire » et donc l'expérience du terrain? Car ces écrits, situés à l'interface de ces deux pôles, génèrent des tiraillements d'ordre discursif provenant d'injonctions paradoxales et de critères d'évaluation pour le moins contradictoires. Nous en citerons ici quelques-uns, issus de nos observations précédentes.

Comment relater une expérience avec toutes les précautions inhérentes à ce type d'activités : recueillir des données, rester objectif, etc. et, en même temps, prendre une certaine « hauteur de vue ", c'est-à-dire, analyser, critiquer ? Distanciation et implication nourrissent la même intention rédactionnelle, mettant ainsi en tension ces écrivants qui, tout à coup, doivent quitter l'expérientiel pour atteindre le réflexif dans une posture de stagiaire qui les oblige d'ailleurs à la plus grande modestie. Préconisations, conseils, perspectives sont abordés du bout de la plume par les uns, plus clairement par les autres, selon les places et missions occupées dans le stage.

41 Pour l'écrivant, la tension provient également de cette nécessité de produire à la fois un écrit relatant l'expérience objectivée, preuve incontournable d'un certain professionnalisme mais aussi un écrit impliquant, preuve de son engagement dans la profession. L'emploi du « je » devient dès lors inconfortable : dire «je » mais pas trop, pour ne pas risquer «la suffisance» (principe de modestie), exister tout en s'effaçant, être sans le faire savoir. Comment passer du visible à l'invisible dans le discours? Mais c'est aussi la relation au « dire » qui est fortement interrogée : dire ce qu'il faut mais juste ce qu'il faut (principe de pertinence) ; dire le maximum de choses mais ne pas tout dire (principe de précaution). C'est donc tiraillé entre ces principes discursifs que l'étudiant doit affirmer son «territoire » et son professionnalisme, tout en conservant une pensée dense dans un écrit fluide (principe de clarté)! Belle prouesse langagière à laquelle, d'ailleurs, il n'a souvent pas été préparé.

42 Exercice de style bien périlleux où s'entrechoquent des registres de discours, ce qui l'amène à jongler entre le descriptif, l'analytique, le narratif, l'explicatif, l'argumentatif, le réflexif, etc. et à rechercher une juste mesure entre la théorie et la pratique, entre le langage de soi et le langage d'autrui (auteurs). D'une certaine façon, il s'agit pour lui de produire un écrit singulier qui étonne, qui aborde une question avec originalité et pertinence, mais en même temps qui reste conforme à l'image du "produit fini », de l'objet observé, de la question analysée. Ainsi, il devra montrer sa capacité à généraliser à partir de situations singulières afin d'atteindre un écrit personnalisé, en lien avec les cadres référentiels souhaités. C'est donc entre singularité et conformité, entre généralité et personnalisation que se joue tout le processus d'écriture.

43 La difficulté est non seulement de trouver la juste mesure dans ce qu'il écrit (gérer le " vouloir écrire »), de dépasser ses compétences scripturales (gérer le "pouvoir écrire » (Reuter, 1996: 72)), mais aussi de manier la rhétorique pour mettre en valeur l'action (gérer le " savoir écrire »), puisque celle-ci semble déterminante dans l'évaluation.

44 Écrire, dans ce cadre, c'est oser quitter ses savoir-faire, et risquer le déséquilibre scriptural. 
Conclusion : des pistes didactiques

Écrire, évaluer un rapport de stage ou un mémoire professionnel, c'est donc être confronté à la fois à des pratiques rédactionnelles et évaluatives complexes, ambivalentes, et en même temps à des représentations fortes issues de constructions individuelles et sociales.

Qu'on le veuille ou non, qu'on en ait conscience ou non, la connaissance des procédés d'écriture et des stratégies discursives facilite la construction de ces productions. C'est pourquoi leur formalisation n'est pas la résultante du seul "savoir écrire " naturel, comme le laisseraient parfois à penser les normes explicites rencontrées dans les ouvrages didactiques liés à ce genre. Atelier d'écriture, aide à l'écriture (Guibert, 2003) sont autant de lieux à développer pour que les étudiants puissent, comme l'évoque Yves Reuter (1996 : 75), conscientiser ces tensions, et penser l'écriture en termes de résolution de problèmes et non seulement en termes de règles et de normes constitutives du genre.

sens, on peut se demander si nos consignes rédactionnelles n'ont pas, elles aussi, à évoluer. Il s'agirait davantage alors de mettre l'accent sur les intentions scripturales, sur les compétences liées à l'activité d'écriture plus que sur le seul produit fini. Par exemple, ne pourrait-on pas associer le genre et sa finalité : «Écrire un rapport de stage » et/ou « décrire et analyser une expérience vécue »?; «Écrire un mémoire professionnel » et/ ou " écrire une situation problème rencontrée »?; "Écrire un mémoire professionnel » et/ou « écrire une réflexion structurée à partir d'une expérience vécue » ?, etc.

Cet aller-retour entre genres et compétences scripturales permettrait à la fois d'éviter des dérives trop formelles qui enferment l'écrivant dans des normes, d'explorer l'hétérogénéité des genres par la conscientisation des intentions scripturales, ellesmêmes hétérogènes, et d'inscrire ces écrits dans une finalité. À partir de ce recadrage, des pistes didactiques apparaitraient plus clairement autour d'objectifs tels que : "écrire pour agir »; « écrire pour comprendre »; « écrire pour rendre compte ». Il est frappant de s'apercevoir, d'ailleurs, comment les enseignants usent de stratégies de contournement pour renommer ces écrits. Ils parlent de "rapport d'étonnement », « de rapport de découverte ", "d'étude-conseil», de "témoignage», de "rapport d'expérience ».

Ce glissement terminologique laisserait-il de côté certaines confusions pour mieux en retrouver d'autres? Cela n'est pas certain. Ne permettrait-il pas, plutôt, d'éclairer différemment les objectifs scripturaux des étudiants? Peut-être aurions-nous alors davantage d'écrivants qui chercheraient à dépasser les cadres formels, pour devenir les vrais auteurs d'une production dont ils seraient plus sûrement les "maitres d'œuvre", " car ces écrits constitueraient, dès lors, pour eux, une façon de se réaliser eux-mêmes à travers ce qu'ils auraient produit, une façon aussi de continuer à se reconnaitre dans leur travail, par-delà son achèvement.» (Le Bouëdec et Tomamichel, 2003 : 193). Peut-être aussi aurions-nous intérêt à clarifier nos modes d'accompagnement, à réfléchir collectivement à nos critères d'évaluation, afin de réguler les tensions dans l'écriture.

Au terme de ce travail, nous souhaitons souligner cet « entre-deux » dans lequel tout écrit professionnel s'inscrit: à la fois processus et produit, dynamique et statique. Mais justement, n'est-ce pas cette formalisation si complexe qui fait de lui un véritable rite de passage? 


\section{BIBLIOGRAPHIE}

Camus, B. (1998) : Rapports de stage et mémoires, Paris, Éditions d'Organisation.

Chaix, M.-L. et Lemey, B. (1994) : Usages de la recherche en situations de formation

professionnelle : le point de vue d'un enseignant-chercheur, Recherche et formation, 17, 9-21.

Cros, F. (1994) : Le mémoire professionnel : un moyen de développement des capacités à acquérir des compétences professionnelles, Recherche et formation, 17, 104-110.

L'Heudé, S. (2005) : Évaluation de mémoire professionnel en IUFM, Questions de pédagogie dans

l'enseignement supérieur, Actes de colloque, École centrale de Lille, 298-302.

Laborde-Milaa, I. (1999) : Écrire un rapport de stage, Paris, Seuil.

Le Bouëdec, G. et Tomamichel, S. (2003) : Former à la recherche en éducation et formation.

Contributions didactiques et pédagogiques, Paris, L'Harmattan.

Maffre, A. (1998) : Réussir son mémoire professionnel, Lyon, Chroniques sociales.

\section{NOTES}

1. <http://cdl.univ-lyon2.fr/article.php3?id_article=3>

2. IUP « Métiers de la formation », dispositif du Département des Sciences de l'éducationLille 1.

\section{RÉSUMÉS}

Rapports de stage et mémoires professionnels sont, dans de nombreux dispositifs de formation, des productions écrites incontournables, parfois enseignées, toujours évaluées. La question de l'accompagnement des étudiants pour ce passage à l'écriture est par ailleurs un sujet qui entraine très souvent des débats animés et contrastés chez les enseignants : comme si le simple fait d'évoquer ces activités d'écriture réveillait des conceptions individuelles bien ancrées et non révisables. Nous avons donc cherché à connaitre les normes et les représentations qui conduisent les pratiques scripturales des étudiants et des évaluateurs à partir de trois corpus distincts et complémentaires. Au terme de ces analyses, nous montrerons que l'activité d'écriture « rapport de stage » et « mémoire professionnel » est porteuse de messages paradoxaux, contribuant ainsi à générer des zones de tension. 